\title{
Categories, Causality, and Numbers: The Problem of Metaphysics in the Critique of Pure Reason
}

\author{
Kazuhiko Yamamoto \\ Megumi Institute of Ethics and Philosophy, Japan
}

\begin{abstract}
We hypothesize that the conundrums in regard to mathematics and quantum mechanics have derived from the same source, i.e., Kant's metaphysics which rests on geometry in virtue of science. It has been found that metaphysica naturalis would be the key to cope with the conundrums in regard to Kant's metaphysics and mathematics.
\end{abstract}

Keywords: Categories, Causality, Geometry, Numbers, Metaphysics

\section{INTRODUCTION}

In regard to quantum mechanics, it is said that "the laws of quantum mechanics dictate that in the absence of measurement, neither of the particles possesses a definite spin until one of the two speeding entangled particles is measured. Once a measurement of one particle is made, the state of the other changes instantaneously, even if the particles are separated by vast distances! Einstein believed this 'spooky action at a distance' was nonsense. His own special theory of relativity held that nothing could travel faster than light, so there was no way for two particles to communicate with each other instantaneously from opposite sides of the universe...We arrive at a paradox, a logical inconsistency...We must be making some mistake - but where? ...Indeed, we might never know the solutions to these puzzles" (HOSSENFELDER 2015: 47-49). When and where humans made mistake is the issue which we try to tackle here, though our inquiry into it is in the stage of hypothesis. We hypothesize that there are two reasons why humans have made mistakes: 1) they abstract from things which appear in the world of sense, and think of the product of the abstraction in the light of geometry: 2) this way of thinking started in the ancient Greek world, indicating that this mistake has a history of more than two millennium. In this regard, if we try to solve this problem, we might have to go back to the world before geometry and mathematics, as a science, appeared among humans, i.e., to primordial times (YAMAMOTO 2017a: 19-37, YAMAMOTO 2017c: 57-70). Since we think that the conundrums in regard to metaphysics, mathematics and physics have originated from the same root of mistake, and that the solution of the conundrums would potentially cause a fundamental change among humans' mentality and psychology, hopefully, for the better, we have begun to try to solve them (YAMAMOTO 2016: 87-100, YAMAMOTO 2017a: 19-37, YAMAMOTO 2017b: 72-81, YAMAMOTO 2017c: 57-70).

\section{Metaphysical Axioms and Mathematical AXioms}

We have already made "synthetic a priori propositions" (B73*) on the ground of metaphysics in the Critique of Pure Reason (YAMAMOTO 2016: 87-100, YAMAMOTO 2017c: 57-70). When it has been found that the synthetic a priori propositions are correspondent to the "universal cognitions $a$ priori" (A300) - "a cognition from a principle" (B357) - we have presented them as metaphysical axioms (YAMAMOTO 2017c: 57-70). Mathematics has been thought to ground in axioms (HILBERT 1902: 437-479, HILBERT 1950: 1-22). However, when Peano makes a proposition in regard to axioms (RUSSELL 1920: 1-12), they seem to exemplify his "synthetic propositions" (A33).

\footnotetext{
* B73 designates the pagination of the standard German edition of Kant's works, as indicated by means of marginal numbers in the Critique of Pure Reason (Kant, Immanuel, Critique of Pure Reason, Cambridge University Press, 1999). All citations are the same.
} 
Do Peano axioms hold true? Provided the "presupposition" (A681/B709) in regard to "the three primitive ideas in Peano's arithmetic...0, number, successor,..." (RUSSELL 1920: 1-12) signifies the "universal cognitions a priori" (A300), these statements should be regarded as axioms. However, when we examine these axioms, we note that these axioms are not commensurable with metaphysical axioms (YAMAMOTO 2017c: 57-70). If we try to dovetail Peano axioms for natural numbers with metaphysical axioms for our "real number: $0, i^{2}=-1$, and 1" (YAMAMOTO 2017b: 72-81, YAMAMOTO 2017c: 57-70), the axioms are to be paraphrased as follows (YAMAMOTO 2017c: 5770).

- Axiom One: 0 is a real number.

- Axiom Two: Every real number has a successor.

- Axiom Three: 0 can be the successor of any real number.

- Axiom Four: Even if the successor of $x$ equals the successor of $y, x$ does not necessarily equal $y$.

- Axiom Five: If a statement is true of 0 , and if the truth of that statement for a number implies its truth for the successor of that number, then the statement is true for every real number.

We think that Peano axioms, which are to be commensurate with metaphysical axioms, can determine something true concerning "the three primitive ideas in Peano's arithmetic...0, number, successor,..." (RUSSELL 1920: 1-12). These axioms indicate that 1) 0 is to signify nullity in space-time - spacetime itself - while number to signify infinite steps of alteration of filled space-elapsing time or its consummation or nullity in space-time; 2) succession is to signify the alteration from nullity in spacetime to a part of filled space-elapsing time or from a part of filled space-elapsing time to empty spacenullified time, i.e., nullity in space-time, suggesting that 1) $i^{2}=-1$ is to signify infinite steps of alteration of space-time itself - quantum - between filled-elapsing and empty-nullified, 2) $0=$ nullity in space-time - space-time itself - is to permeate filled space-elapsing time, 3) $i^{2}=-1$ is to come across its cessation any time before the consummation of itself as number 1 (YAMAMOTO 2017c: 57-70).

Why does the contrariety between mathematical axioms and metaphysical axioms - the problem for mathematics - occur? We think that the root of the problem for mathematics resides in Kant's metaphysics (YAMAMOTO 2017a: 19-37, YAMAMOTO 2017b: 72-81, YAMAMOTO 2017c: 5770). What kind of problem do mathematics and Kant's metaphysics have in common? It is geometry, on which Kant's metaphysics rests. Kant's so-called antinomy of pure reason in the system of cosmological ideas derives from geometry (YAMAMOTO 2017b: 72-81). In order to solve the problem of antinomy of pure reason, Kant has to think of the "regulative principle of reason" (A517/B545) and his "thing in itself" (YAMAMOTO 2016: 87-100, YAMAMOTO 2017a: 19-37). However, "thing in itself" is thought to signify an epistemological naught. Why do "all propositions of geometry" (A47) lead Kant to the antinomy of pure reason? In geometry, this kind of thing happens: "with two straight lines no space at all can be enclosed, thus no figure is possible, and try to derive it from the concept of straight lines and the number two; or take the proposition that a figure is possible with three straight lines, and in the same way try to derive it from these concepts. All of your effort is in vain, and you see yourself forced to take refuge in intuition, as indeed geometry always does" (B65-A48). Kant, who says that "geometry is a science that determines the properties of space synthetically and yet a priori. What then must the representation of space be for such a cognition of it to be possible? It must originally be intuition; for from a mere concept no propositions can be drawn that go beyond the concept, which, however, happens in geometry" (B40-B41), seems to have firmly believed in geometry while harboring an indelible doubt about it on account of the fact that it seems impossible to derive a figure from the concept of straight lines and the number two. Since "empirical intuitions" (A229) or "empirical synthesis" or "empirical cognition" (A229) must be entirely separated from a thing in itself in Kant's metaphysics because of ethics (YAMAMOTO 2016: 87100), geometry lures Kant to think of "the properties of space synthetically and yet a priori" (B40) "its transcendental ideality" (A28) - according to the geometrical way of thinking (YAMAMOTO 
2017a: 19-37, YAMAMOTO 2017b: 72-81, YAMAMOTO 2017c: 57-70). However, when Kant noticed that geometry incessantly leads him to the abyss of the antinomy of pure reason, he, who thinks that the transcendental ideality of space is "nothing as soon as we leave out the condition of the possibility of all experience, and take it as something that grounds the things in themselves" (A28), cannot but try to solve the conundrum by means of thinking the "regulative principle of reason" and his "thing in itself" as the condition of the transcendental ideality of space in place of geometry whose "condition of the possibility of all experience" is already left out (YAMAMOTO 2016: 87-100, YAMAMOTO 2017a: 19-37). We think that one cannot attain to space itself through geometry since it has a fundamental defect. When Kant reveals his way of thinking in regard to geometry, saying, "this intuition must be encountered in us a priori, i.e., prior to all perception of an object, thus it must be pure, not empirical intuition" (B41), we have to say, in an opposite manner, that 1) we must encounter an object a priori prior to all perception of an object; 2) then we will perceive an object through pure intuition affected by the sensation of nullity - empirical intuition - and synthesis of apprehension. What is this object? It is an object itself, i.e., death itself - nullity in space-time. On the contrary, Kant's "thing in itself" (A676/B704) is meant to be an epistemological naught, as he himself repeatedly implies, saying, "One mistakes the significance of this idea right away if one takes it to be the assertion, or even only the presupposition, of an actual thing to which one would think of ascribing the ground for the systematic constitution of the world; rather, one leaves it entirely open what sort of constitution in itself this ground, which eludes our concepts, might have,..." (A681/B709). If one tries to ground metaphysics in epistemological naught, it is absolutely wrong. We must ground metaphysics in the transcendental analytic (YAMAMOTO 2016: 87-100, YAMAMOTO 2017a: 19-37).

We can solve the problem derived from geometry by means of cognizing nullity in space-time - death itself - through pure intuition affected by the sensation of nullity - empirical intuition - and synthesis of apprehension (YAMAMOTO 2016: 87-100, YAMAMOTO 2017a: 19-37), which would enable us to attain the pure concepts of the understanding or synthetic a priori cognition. Since, in "all propositions of geometry," there are always points or lines or numbers along with nullity in spacetime, transcendental ideality of space and time always comprises points or lines or numbers. The cohabitation of nullity with points or lines or numbers in Kant's transcendental ideality of space and time leads Kant to the conundrum - the antinomy of pure reason. He has to erase points or lines or numbers in the transcendental ideality of space and time drawn from geometry. Kant explicate how to solve it, saying, "as far as concerns the void that one might think of outside of the field of possible experience (the world), this does not belong to the jurisdiction of the mere understanding, which only decides about questions concerning the use of given appearances for empirical cognition, and it is a problem for ideal reason, which goes beyond the sphere of a possible experience and would judge about what surrounds and bounds this, and must therefore be considered in the transcendental dialectic" (A229-B282). Thus, Kant's solution is to rely on "transcendental dialectic" (in other words, merely thinking of a thing in itself). Kant himself explain why he resorts to transcendental dialectic, saying, "The principle of continuity forbade any leap in the series of appearances (alterations) (in mundo non datur saltus), but also any gap or cleft between two appearances in the sum of all empirical intuitions in space (non datur hiatus)" (B281-A219). This is absolutely wrong. Humans are to meet nullity in space-time in experience or in possible experience, which would enable them to reach "the principle of continuity" through empirical intuition or empirical synthesis or empirical cognition. We do not have to seek the encounter. It comes to us on its own necessarily and universally. What is this? It is death itself - disappearance of what appeared. We have to think that 1) appearance signifies filled space-elapsing time while the disappearance of what appeared signifies empty space-nullified time, i.e., nullity in space-time, 2) nullity in space-time - space-time itself permeates filled space-elapsing time. We think that it is nullity in space-time that would enable humans to cognize appearances in filled space-elapsing time. This is our transcendental ideality of space-time - "transcendental ideality of appearances" (A506/B534). We need not resort to the "transcendental dialectic" in regard to the issues of "the principle of continuity" (B281) and "the 
void" (A229) since we can think of the void - nullity in space-time - inside "of the field of possible experience (the world)" (A229).

Since our "universal propositions" (B358) - metaphysical axioms - signify "universal cognitions $a$ priori," they can serve as the major premise in a syllogism.

a. Our metaphysical axioms - "a cognition from a principle" (B357) - signifies "the category (which constitutes its unity) insofar as it is universal and rests on a rule a priori" (A138/B177-A178).

b. "Every syllogism is a form of derivation of a cognition from a principle" (B357). When "a cognition from a principle" belongs among the categories, it corresponds to the "categorical syllogisms" (A406). Therefore, "a cognition from a principle" - nullity in space-time - works as the major premise in categorical syllogisms, stating "the relation of a predicate to a subject" (B433), i.e., that nullity in space-time - space-time itself - permeates a subject.

c. Since the major premise - nullity in space-time - "always gives a concept such that everything subsumed under its condition can be cognized from it according to a principle" (B357), humans' cognition can ground in nullity in space-time, indicating that it has "the reality (i.e., objective validity)" (A28) of space and time, which is necessary and universal. Kant says in regard to "the reality (i.e., objective validity)" of time, that "time is a necessary representation that grounds all intuition....In it alone is all actuality of appearances possible" (A31), suggesting that time itself is "other subjective representation related to something external that could be called a priori objective" (A28).

What is the major premise in regard to Kant's metaphysics or mathematics? The answer is that while it is Kant's "thing in itself" in regard to his metaphysics, it is "all propositions of geometry" in regard to mathematics. What kind of problem happens in Kant's metaphysics or in mathematics? As indicated above, in Kant's metaphysics: "thing in itself" - mere thought - signifies an epistemological naught while in mathematics: there are always points or lines or numbers along with nullity. We think that, if nullity in space-time is introduced in accordance with "an a priori intuition" (B40) or "pure intuition" (A21), it would neutralize the conundrum, which unavoidably occurs in "all propositions of geometry." It means that space can be enclosed with two straight lines and a figure is possible, if "figures in space" are under the aegis of "pure a priori imagination" (A142). The so-called "imaginary number" $i^{2}=-1$ in mathematics can be thought to pertain to "pure a priori imagination" or "pure a priori intuition" (A77). In our metaphysics, the imaginary number $i^{2}=-1$ is commensurate with "one that is also merely empirical, i.e., a proposition of experience" (A47), which can "contain necessity and absolute universality." In this regard, the "imaginary number" $i^{2}=-1$ is regarded to be a "real number," while a "real number" in mathematic is to be an "imaginary number" except 0 or 1 (YAMAMOTO 2017b: 72-81, YAMAMOTO 2017c: 57-70). We think that Peano axioms, in commensurate with metaphysical axioms, indicate that 1) $i^{2}=-1$ is to signify infinite steps of alteration of space-time itself, 2) $0=$ nullity in space-time - space-time itself - is to permeate filled space-elapsing time, which is on the way of infinite steps of alteration to its consummation as number 1. Since our "real numbers $0, i^{2}=-1$, and 1 " are supposed to signify "everything real in appearance" (B214) which "has for the same quality its degree (of resistance or of weight) which, without diminution of the extensive magnitude or amount, can become infinitely smaller until it is transformed into emptiness and disappears" (B216), we can make more axiomatic propositions in regard to these numbers that 1) the property which belongs to 0 , namely nullity in space-time is followed by the property which belongs to "imaginary number" $i^{2}=-1$, namely filled space-elapsing time - quantum 2 ) when the property which belongs to "imaginary number" $i^{2}=-1$ attains the property which belongs to 1 , it plunges into nullity in space-time, i.e., into the property which belongs to 0 (YAMAMOTO 2017c: 57-70).

\section{Categories, Causality and Numbers}

Furthermore, we can deduce from Peano-metaphysical axioms more "universal propositions" as follows (YAMAMOTO 2017c: 57-70): 
a. All alteration as a transition of a thing from one state to another signifies: 1) an alteration of a part of filled space-elapsing time to empty space-nullified time: 2) a passing out of a part of filled space-elapsing time into a part of filled space-elapsing time as the succession of the states itself: 3) an alteration of empty space-nullified time to a part of filled space-elapsing time.

b. It is impossible for a part of filled space-elapsing time and another part of filled space-elapsing time to be at the same point in the same instance.

c. It is possible for a part of empty space-nullified time and another part of empty space-nullified time to be at the same point in the same instance - nullity in space-time - if points and instances are conjured up in nullity in space-time.

d. The form of appearance, which alterability concerns, is filled-elapsing or empty-nullified, while their cause is in the unalterable - space-time itself.

e. All appearances arise in a spontaneity, which could start to act from itself, without needing to be preceded by any other cause that in turn determines it to action according to the law of causal connection.

f. The synthesis of the manifold part of space-time itself is successive, and thus contains a series.

g. The synthesis of the manifold part of space-time itself takes place in the manifold of sensibility filled space-elapsing time.

h. Succession, subordination and coordination which take place in filled space-elapsing time affect the world-whole.

These metaphysical axioms would provide us a clue to comprehending "the principle of causality" (A247) by means of "the Transcendental Analytic" (A246/B303), which, in an opposite manner to what Kant refers to (A246/B303-A247), has these important results: either that "the understanding can never accomplish a priori anything more than to anticipate an object of experience or possible experience in general, and, since that which is not appearance itself cannot be an object of experience or possible experience, it can never overstep the limits of sensibility, within which alone objects in themselves are given to us" (YAMAMOTO 2017a: 19-37) or that "the understanding can never accomplish empirically anything more than to anticipate the form of experience or possible experience in general, and, since that which is not appearance itself cannot be the form of experience or possible experience, it can never overstep the limits of sensibility, within which alone the form is given to us." We think that since 1) "the laws of appearances in nature" (B164) agree "with the understanding and its a priori form, i.e., its faculty of combining the manifold in general" (B164) and 2) "appearances themselves" agree "with the form of sensible intuition a priori" (B164), an object of experience or possible experience which the understanding can anticipate a priori and the form of experience or possible experience which the understanding can anticipate empirically correspond to "categories." Since, in our transcendental analytic, an object of experience or possible experience, i.e., death itself is homogeneous with the form of experience or possible experience, i.e., nullity in spacetime, metaphysical axioms are to signify the "transcendental deduction" (B159), in which the "possibility as a priori cognitions of objects of an intuition in general was exhibited" (B159), spawning "the possibility of cognizing a priori through categories whatever objects may come before our senses" (B159). We think that "natural or real numbers" in mathematics signify an object of possible experience which the understanding can anticipate a priori or the form of possible experience which the understanding can anticipate empirically. We have to note that mathematical axioms and natural or real numbers in mathematics, which rest on geometry, state something pertaining to an $a$ priori anticipation of an object of possible experience or empirical anticipation of the form of possible experience, while metaphysical axioms and our "real numbers $0, i^{2}=-1$, and 1" (YAMAMOTO 2017b: 72-81, YAMAMOTO 2017c: 57-70), which rest on the law of nature, state something pertaining to an a priori anticipation of an object of experience, or empirical anticipation of the form of experience. Furthermore, we say that since our "real numbers $0, i^{2}=-1$, and 1 " are to pertain to "the 
continuum" which "has the next cardinal number beyond that of the countable assemblage," they signify "the cardinal number of the continuum" (HILBERT 1902: 437-479). Therefore, it can be said that our "cardinal numbers $0, i^{2}=-1$, and 1 " are to be "real numbers," while natural or real numbers in mathematics except 0 or 1 are to be "imaginary numbers" (YAMAMOTO 2017b: 72-81, YAMAMOTO 2017c: 57-70).

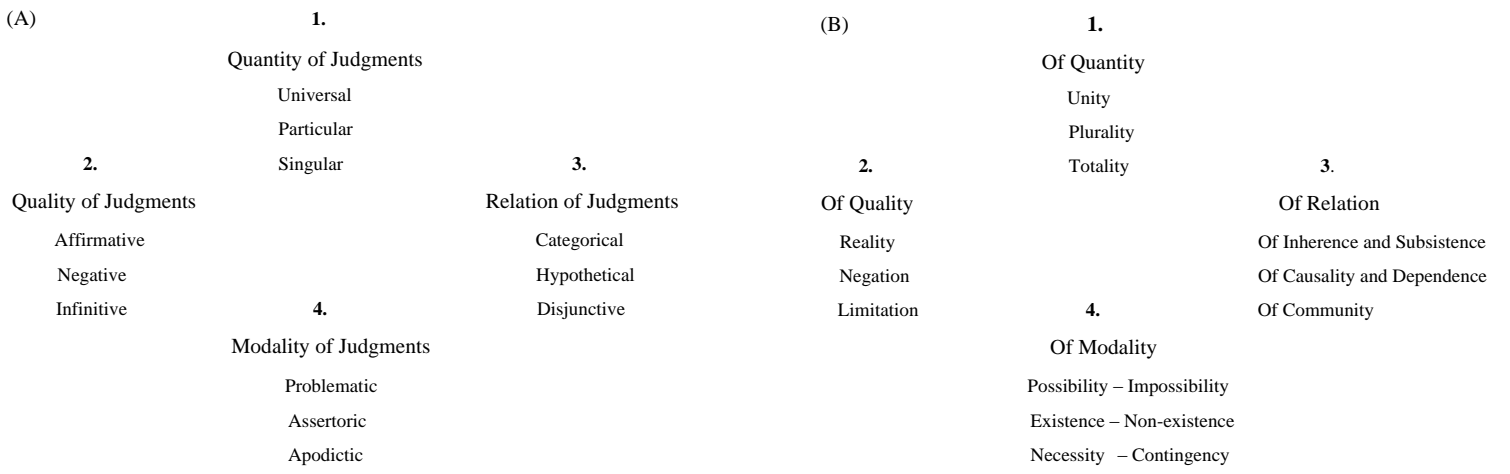

Figure 1. The scheme which, Kant thinks, is the clue to the discovery of all pure concepts of the understanding.

(A) Table of Logical Functions. (B) Table of Categories.

When natural or real numbers in mathematics are thought to pertain to an object of possible experience or the form of possible experience, they might be empty provided the "possible experience" and the understanding remain contingent. In order to prop up natural or real numbers in mathematics, Kant has to resort to "logical functions of thinking" (B159) and "all categories" which "are grounded on logical functions in judgments" (B131). Kant seems to expect that the "logical functions of thinking" and "all categories" which "are grounded on logical functions in judgments" would give him "the clue to the discovery of all pure concepts of the understanding" (A70/B95) numbers. However, when Kant separates "an object of experience which the understanding can anticipate a priori" from "the form of experience which the understanding can anticipate empirically," he cannot but resort to "transcendental dialectic (in other words, merely thinking of a thing in itself)." This is wrong. We have to think that "thing in itself" pertains to "an object of experience or possible experience which the understanding can anticipate a priori and the form of experience or possible experience which the understanding can anticipate empirically." The scheme in Fig.1, which is expected to be the clue to the discovery of all pure concepts of the understanding, is empty on account of the fact that Kant's "thing in itself" is thought to be an epistemological naught. On the contrary, metaphysical axioms teach that "an object of experience or possible experience which the understanding can anticipate a priori" and "the form of experience or possible experience which the understanding can anticipate empirically" is nothing but nullity in space-time, i.e., space-time itself. Only on this cognition, the "Table of Categories" (B106) in Fig. 1 is to become "uncommonly useful, indeed indispensable in the theoretical part of philosophy for completely outlining the plan for the whole of a science insofar as it rests on a priori concepts, and dividing it mathematically in accordance with determinate principles,..." (B109). When Kant reveals his idea concerning the Table of Categories, saying, "this table, which contains four classes of concepts of the understanding, can first be split into two divisions, the first of which is concerned with objects of intuition (pure as well as empirical), the second of which, however, is directed at the existence of these objects (either in relation to each other or to the understanding). I will call the first class the mathematical categories, the second, the dynamical ones" (B110), we think that Kant's way of thinking that "the first class the mathematical categories, the second, the dynamical ones" is wrong. When Kant says, "insofar as it rests on a priori concepts, and dividing it mathematically in accordance with determinate principles,..." (B109), we have to say that the first class belongs among the dynamical categories or metaphysical categories, while the second are the mathematical ones. Why Kant has made this mistake can be seen in his saying, "As one sees, the first class has no correlates, which are to be met 
with only in the second class. Yet this difference must have a ground in the nature of the understanding" (B110). On the contrary to Kant, we see that both the first class and the second class have correlates: nullity in space-time - space-time itself (YAMAMOTO 2017a: 19-37, YAMAMOTO 2017b: 72-81, YAMAMOTO 2017c: 57-70). Therefore, there is no difference "in the nature of the understanding" between the first class and the second class. In our metaphysics, "the nature of the understanding" is commensurate with understanding the correlates in the first class and the second class by means of cognizing nullity in space-time through pure intuition affected by the sensation of nullity - empirical intuition - and synthesis of apprehension (YAMAMOTO 2017a: 19-37). "This difference" (B110) appears to have a ground in the way of thinking: a way of thinking resorting to "logical functions of thinking" and "all categories" which "are grounded on logical functions in judgments - metaphysical deduction (B159) - or a way of thinking resorting to "logical functions of thinking" and "all categories" which are grounded on transcendental analytic - transcendental deduction (B159). However, since "all categories" are to be grounded on transcendental analytic (the understanding) and deduction (logical functions of thinking), "this table, which contains four classes of concepts of the understanding" (B110), does not have "this difference" but appears to have "this difference."

Kant explains why the Table of Logical Functions in Figure 1 is indispensable for "the discovery of all pure concepts of the understanding" (A70/B95), saying, "The manifold that is given in a sensible intuition necessarily belongs under the original synthetic unity of apperception, since through this alone is the unity of the intuition possible. That action of the understanding, however, through which the manifold of given representations (whether they be intuitions or concepts) is brought under an apperception in general, is the logical function of judgments. Therefore all manifold, insofar as it is given in one empirical intuition, is determined in regard to one of the logical functions for judgment, by means of which, namely, it is brought to a consciousness in general. But now the categories are nothing other than these very functions for judging, insofar as the manifold of a given intuition is determined with regard to them. Thus the manifold in a given intuition also necessarily stands under categories" (B143). From our viewpoint, these enigmatic remarks indicate four things; 1) the manifold that is given in a sensible intuition necessarily belongs under "consciousness of itself (apperception)" (B68) and the apperception necessarily stands under the law of nature, 2) since the manifold of given representations - intuitions or concepts - stand under an apperception in general, and an apperception performs the action of the understanding, intuitions or concepts stand under the law of nature and "a perception itself" (A180) endowed with the faculty of "the synthetic a priori cognition" (A14/B28), 3) since "the synthetic a priori cognition" is brought to a consciousness in general through the logical function of judgments, all manifold, insofar as it is given in one empirical intuition, i.e., nullity in space-time or space-time itself, is determined in regard to "a perception itself" endowed with the faculty of "the synthetic a priori cognition," by means of the logical functions for judgment, 4) since all manifold, insofar as it is given in one empirical intuition, belong among "a perception itself" endowed with the faculty of "the synthetic a priori cognition" - categories - the manifold in a given intuition also necessarily stands under categories. We asserts that "categories," which are to arise through the function of the synthetic a priori cognition within "the standing and lasting I (of pure apperception)" (A123) or "consciousness of itself (apperception)" (B68), are the determination of the manifold of a given intuition, leading the original unity of apperception to the understanding, through the logical function of judgments, that the manifold of a given intuition exists as objects themselves. When "the manifold in a given intuition also necessarily stands under categories" (B143), can we say that the manifold of a given intuition, which exists as an object, also necessarily stand under numbers?

When Kant says in regard to the Table of Categories that "allness (totality) is nothing other than plurality considered as a unity, limitation is nothing other than reality combined with negation, community is the causality of a substance in the reciprocal determination of others, finally necessity is nothing other than the existence that is given by possibility itself" (B111), and furthermore says in regard to number that "the concept of a number (which belongs to the category of allness) is not always possible wherever the concepts of multitude and of unity are" (B111), we have to say, in an 
opposite manner to him, that "the concept of a number (which belongs to the category of allness) is always possible since allness (totality) is nothing other than plurality considered as a unity and countable as a number." Here, a unity must be thought to be a part of nullity in space-time - a part of space-time itself - under the condition that it is possible for "the standing and lasting I (of pure apperception)" (A123) or "consciousness of itself (apperception)" (B68) to conjure up a priori the limitation for space-time itself. Is it possible? Yes, it is possible, which Kant suggests, saying, "The infinitude of time signifies nothing more than that every determinate magnitude of time is only possible through limitations of a single time grounding it. The original representation, time, must therefore be given as unlimited" (A32-B48). While nullity in space-time, i.e., space-time itself signifies "the a priori concepts of space and time" (B57), which are not "creatures of the imagination, the origin of which must really be sought in experience, out of whose abstracted relations imagination has made something that, to be sure, contains what is general in them, but that cannot occur without the restrictions that nature has attached to them" (B57), a part of nullity in space-time or a part of space-time itself - "the schema of sensible concepts (such as figures in space)" (B181-A142) - is "a monogram of pure a priori intuition" (A142) or a product "of the imagination, the origin of which must really be sought in experience, out of whose abstracted relations imagination has made something that, to be sure, contains what is general in them, but that cannot occur without the restrictions that nature has attached to them" (B57). While nullity in space-time or space-time itself is to be cognized upon encountering a disappearance of a thing which appeared, a part of nullity in space-time or a part of space-time itself - "the schema of sensible concepts (such as figures in space)" (B181-A142) - can be thought to lie within the field of possible experience. Since "nowhere beyond the field of possible experience can there be any synthetic a priori principles" (A248-B305), and all of cognitions in regard to our "cardinal numbers $0, i^{2}=-1$, and 1 " and natural or real numbers in mathematics can be thought to "lie in the entirety of all possible experience" (A146), we would say that numbers, including our "cardinal numbers $0, i^{2}=-1$, and 1 " and "natural or real numbers" in mathematics," signify "synthetic a priori principles" (B305). When Kant says in regard to numbers, "The pure schema of magnitude (quantitatis), however, as a concept of the understanding, is number, which is a representation that summarizes the successive addition of one (homogenous) unit to another. Thus number is nothing other than the unity of the synthesis of the manifold of a homogeneous intuition in general" (B182-A143), we entirely agree with him, thinking that the "pure schema of magnitude (quantitatis)" or "(homogeneous) unit" - number - signifies a figure among nullity in space-time, whose image is conjured up in humans' brain in virtue of "mathematicaltranscendental ideas" (A529/B557). Since "appearances themselves" (A104) - empty space-nullified time or filled space-elapsing time - is commensurate with "the transcendental ideality of appearances" (A506/B534), they would last forever irrespective of the existence of humans on this planet. In regard to "mathematical axioms" (A300) - numbers - we have already said that "since "nowhere beyond the field of possible experience can there be any synthetic a priori principles' (A248-B305), 'all of our cognitions' (A146), which 'lie in the entirety of all possible experience' (A146), can be enhanced to 'synthetic a priori principles' (B305), i.e., 'transcendental truth, which precedes all empirical truth and makes it possible' (A146)" (YAMAMOTO 2017c: 57-70). In addition, we would say that "all of our cognitions," which can be enhanced to "synthetic a priori principles," belong among categories.

Since a likeness of things which appear in the world of sense or "an analogy of experience" (A180) can be "assumed as a quantum discretum, the multiplicity of units in it is determined; hence it is always equal to a number" (A527/B555). Is a number, which is equivalent to a figure among nullity in space-time - "a concept of the understanding" or "the pure schema of magnitude (quantitatis)" different from a number, which is supposed to be equivalent to "a likeness of things which appear in the world of sense" or "the multiplicity of units" in something which can be assumed as a quantum discretum? No, they are not different, but the same. The former number - "a representation that summarizes the successive addition of one (homogenous) unit to another" (B182) - signifies quantum continuum (A527/B555) in terms of nullity in space-time, while the latter number, which signifies quantum continuum, appears to be quantum discretum under the assumption that a thing signifies 
quantum discretum. We must say that a number, i.e., quantum continuum is homogeneous with "the appearance as quantum continuum" (A527/B555) - a number. However, when geometry and mathematics, as a science, have appeared among humans, inserting points or lines or numbers into nullity in space-time (EUCLID 2002:1-36), a number - quantum continuum - has been alienated from "the appearance as quantum continuum" (A527/B555). Natural numbers in primordial times categories - have ceased to signify "the transcendental ideality of appearances," resulting in lapsing into "numerical formulas" (B206) - the "transcendental principle of the mathematics of appearances" (B206). We think that the conundrums in regard to mathematics and Kant's metaphysics originate in this alienation of numbers from "the transcendental ideality of appearances" while this alienation enabled humans to metamorphose "natural numbers in primordial times" - categories - into "natural or real numbers" in mathematics, enhancing them to "synthetic a priori principles" (B305), i.e., "transcendental truth, which precedes all empirical truth and makes it possible" (A146). However, because Kant annuls "mathematical-transcendental ideas" (A529/B557) by means of the "transcendental dialectic" (B282), it follows that "a likeness of things which appear in the world of sense" or "the multiplicity of units" cannot be assumed as quantum discretum any more. We think that insofar as the "mathematical-transcendental ideas" rest on Kant's "thing in itself" (A676/B704), "natural or real numbers" in mathematics cannot have the properties of categories. "Numerical formulas" (B206) cease to signify the "transcendental principle of the mathematics of appearances" (B206). Here, "pure reason" which "has no other aim than the absolute totality of synthesis on the side of conditions" (A336) or "reason" which "has nothing to do with absolute completeness from the side of the conditioned" (A336) intrudes, and tries to rescue them. Since a number - a figure among nullity in space-time, whose image is conjured up in humans' brain - can be enhanced to "regulative principle of reason" (A517/B545) by means of the function of "reason" which "relates itself only to the use of the understanding...in order to prescribe the direction toward a certain unity of which the understanding has no concept" (B383), "numerical formulas" would last as a likeness of things which appear in the world of sense or "an analogy of experience" (A180) insofar humans exist on this planet. Furthermore, we would say that once numbers including our "cardinal numbers $0, i^{2}=-1$, and 1 " and natural or real numbers in mathematics have been set to ground in transcendental analytic, they would last forever as "synthetic a priori principles," i.e., "transcendental truth" irrespective of the existence of humans on this planet. We think that while our "cardinal numbers $0, i^{2}=-1$, and 1 " are to stand under "transcendental deduction" (B159), natural or real numbers in mathematics are to stand under "transcendental deduction" (B159) and "metaphysical deduction" in which "the origin of the $a$ priori categories in general was established through their complete coincidence with the universal logical functions of thinking" (B159). Therefore, what Kant says in regard to "the antinomy of pure reason in its cosmological ideas" (A506/B534) can be rephrased, in an opposite manner, as follows: "one cannot draw from this antinomy a utility, dogmatic or critical and doctrinal utility, namely that of thereby proving indirectly the transcendental ideality of appearances, since someone has enough in the direct proof in the Transcendental Aesthetic - space-time itself. The proof would not consist in this spurious dilemma."

Following the scheme in the Table of Categories, which ordains that "community is the causality of a substance in the reciprocal determination of others, finally necessity is nothing other than the existence that is given by possibility itself" (B111), we have to deal with the issue of "community (reciprocity)" (A144) in "allness (totality)," i.e., "plurality considered as a unity" (B111) since "the schema of community (reciprocity), or of the reciprocal causality of substances with regard to their accidents, is the simultaneity of the determinations of the one with those of the other, in accordance with a general rule" (A144-B184). Kant's discourse would lead us to an astonishing finding in regard to the issue of causality. Accordingly he says, "since the parts of space are not subordinated to one another but are coordinated with one another, one part is not the condition of the possibility of another, and space, unlike time, does not in itself constitute a series. Yet the synthesis of the manifold parts of space, through which we apprehend it, is nevertheless successive, and thus occurs in time and contains a series" (B439). From our viewpoint, this enigmatic remark indicates seven things: 1) since there are no parts of space in space itself - nullity in space - space, as space itself, is not 
"subordinated to one another" and are not "coordinated with one another" (B439): 2) therefore, space, as space itself, does not constitute a series: 3 ) since there are filled spaces as manifold parts of space itself, the manifold parts of space itself are subordinated to one another or are coordinated with one another: 4) therefore, a filled space - a manifold part - can be the condition of the possibility of another part, and filled space, like elapsing time, does in itself constitute a series: 5) the synthesis of the manifold part of space itself (synthesis of filled space or of a filled space and empty space) is "successive, and thus occurs in time and contains a series" (B439): 6) since the synthesis takes place in the manifold of sensibility, succession, subordination and coordination pertain to filled spaceelapsing time: 7) since the world-whole consists of all appearances - filled space-elapsing time and nullity in space-time - succession, subordination and coordination which take place in filled spaceelapsing time would affect the world-whole. Following what Kant says (B112), contrarily, we have to say that "a similar connection is thought of in an entirety of things, since one is subordinated, as effect, under another, as the cause of its existence, or coordinated with the other simultaneously and reciprocally as cause with regard to its determination." What does this mean? It means that "the members of the division exclude each other and yet are connected in one sphere, so in the latter case the parts are represented as ones to which existence (as substances) pertains to each exclusively of the others, and which are yet connected in one whole" (B113). We think that "the members of the division" signifies categories, through which it would become possible for us to cognize "whatever objects may come before our senses, ... as far as the laws of their combination are concerned" (B159). This metaphysical causality must be the ground on which mathematical axioms rest. We feel that mathematical axioms, in commensurate with metaphysical axioms, would be the clue to solve the conundrum in regard to Hilbert's mathematical problems posed in 1900 (HILBERT 1902: 437-479). Apparently, our "cardinals $0, i^{2}=-1$, and 1" show a resemblance to the issue of Cantor's "transfinite numbers" (CANTOR 1955: 85-201) and "the cardinal number of the continuum" (HILBERT 1902: 437-479) or Riemann zeta function and prime numbers (RIEMANN 2005: 865-876, RIEMANN 2005: 876-885). The conundrums in regard to mathematics (HILBERT 1902: 437-479) and quantum mechanics (EINSTEIN et al. 1935: 777-780) seem to have derived from the same source, i.e., Kant's metaphysics which rests on geometry in virtue of science. We think that "metaphysica naturalis" (B21) would be the key to cope with the conundrums in regard to Kant's metaphysics and mathematics. When Kant, in all likelihood having the "Leibnizian monadology" (A274) or "Leibniz's famous doctrine of space and time" (A275) in mind, criticizes the philosopher, saying, "only the philosopher expresses himself somewhat more determinately in saying that in all alterations in the world the substance remains and only the accidents change. But I nowhere find even the attempt at a proof of this so obviously synthetic proposition, indeed it only rarely stands, as it deserves to, at the head of the pure and completely a priori laws of nature. In fact the proposition that substance persists is tautological" (A184), we would say that "in all alterations in the world the substance remains and only the accidents change in conjunction with the substance. We find a proof of this so obviously synthetic a priori proposition, indeed it only stands, as it deserves to, at the head of the pure and completely a priori laws of nature. In fact the proposition is that substance persists tautologically." We believe that this so obviously synthetic a priori proposition, which stands at the head of the pure and completely a priori laws of nature, corresponds to metaphysica naturalis.

\section{REFERENCES}

Cantor, Georg, Contributions to the Founding of the Theory of Transfinite Numbers, Dover Publications, 1955.

Einstein, Albert, Podolsky, Boris, Rosen, Nathan, "Can Quantum-Mechanical Description of Physical Reality Be Considered Complete?," Physical Review 47: 777-780, 1935.

Euclid, Euclid's Elements, Green Lion Press, 2002.

Hilbert, David, Foundations of Geometry, The Open Court Publishing Company, 1950.

Hilbert, David, "Mathematical Problems," Bulletin of the American Mathematical Society 8: 437-479, 1902.

Hossenfelder, Sabine, "Head Trip: Einstein's Thought Experiments Left a Long and Somewhat Mixed Legacy of Their Own," Scientific American, September 2015, pp. 47-49, 2015. 
Kant, Immanuel, Critique of Pure Reason, Cambridge University Press, 1999.

Riemann, Georg Friedrich Bernhard, "On the Hypotheses Which Lie at the Bases of Geometry," God Created the Integers, Running Press, pp. 865-876, 2005.

Riemann, Georg Friedrich Bernhard, "On the Number of Prime Numbers Less Than a Given Quantity," God Created the Integers, Running Press, pp. 876-885, 2005.

Russell, Bertrand, Introduction to Mathematical Philosophy, The Macmillan, 1920.

Yamamoto, Kazuhiko, "The Pure Concepts of the Understanding and Synthetic A Priori Cognition: the Problem of Metaphysics in the Critique of Pure Reason and a Solution," Proceedings of the European Conference on Ethics, Religion \& Philosophy 2016, pp 87-100, 2016.

Yamamoto, Kazuhiko, "The Transcendental Aesthetic and Absolute Totality of Conditions: The Problem of Metaphysics in the Critique of Pure Reason and a Solution," International Journal of Humanities Social Sciences and Education 4 (2): 19-37, 2017 a.

Yamamoto, Kazuhiko, "Absolute Totality, Causality, and Quantum: The Problem of Metaphysics in the Critique of Pure Reason," International Journal of Humanities Social Sciences and Education 4 (4): 72-81, $2017 \mathrm{~b}$.

Yamamoto, Kazuhiko, "Numbers, Geometry, and Mathematical Axioms: The Problem of Metaphysics in the Critique of Pure Reason," International Journal of Humanities Social Sciences and Education 4 (6): 57-70, $2017 \mathrm{c}$.

Citation: Kazuhiko Yamamoto. "Categories, Causality, and Numbers: The Problem of Metaphysics in the Critique of Pure Reason". International Journal of Humanities Social Sciences and Education (IJHSSE), vol 4, no. 8, 2017, pp. 19-29. doi: http://dx.doi.org/10.20431/2349-0381.0408003.

Copyright: () 2017 Authors. This is an open-access article distributed under the terms of the Creative Commons Attribution License, which permits unrestricted use, distribution, and reproduction in any medium, 